Supporting Information for

\title{
3-Hydroxy-4-nitrobenzoic acid as a MALDI matrix for In-Source Decay
}

\author{
Yuko Fukuyama, ${ }^{1}$ Shunsuke Izumi, ${ }^{2}$ and Koichi Tanaka ${ }^{l}$
}

1. Koichi Tanaka Mass Spectrometry Research Laboratory, Shimadzu Corporation, 1, NishinokyoKuwabaracho, Nakagyo-ku, Kyoto 604-8511, Japan

2. Department of Mathematical and Life Sciences, Graduate School of Science, Hiroshima University, 1-3-1 Kagamiyama, Higashi-Hiroshima 739-8526, Japan

\section{CORRESPONDING AUTHOR}

Yuko Fukuyama

e-mail: yukof@shimadzu.co.jp

Table of Contents:

Figure S-1. ISD spectra of BSA using 3H4NBA in linear positive-ion mode.

Figure S-2. $\quad$ MS and ISD spectra of amyloid $\beta$ 1-40 using 3H4NBA and 1,5-DAN matrices solution prepared on the day, or four months ago, in linear positive-ion mode.

Figure S-3. Mass spectra of 3H4NBA matrix with or without analytes in positive- and negativeion modes. 

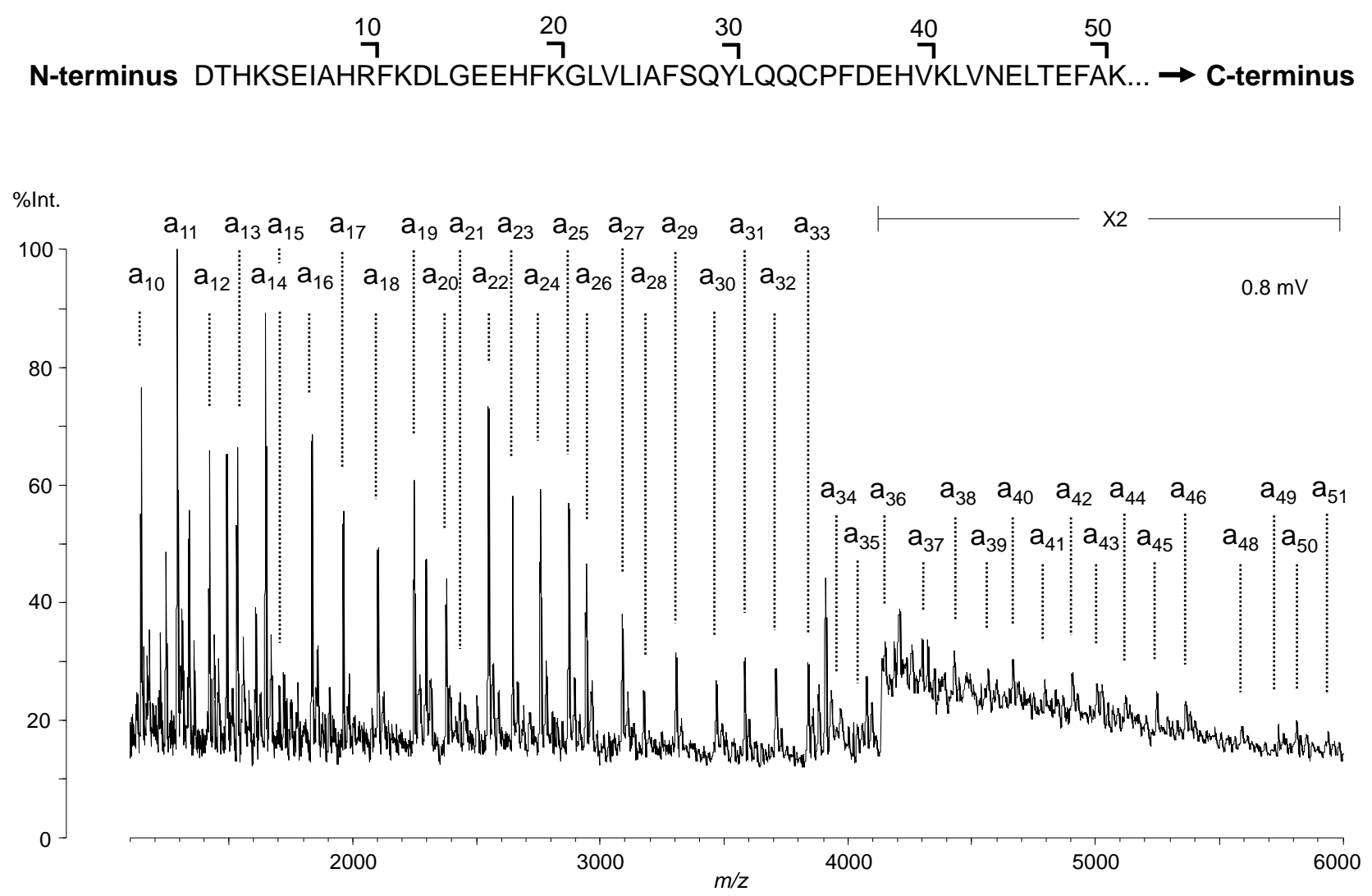

Figure S-1. ISD spectra of BSA using 3H4NBA in linear positive-ion mode. 
ISD

(a) $3 \mathrm{H} 4 \mathrm{NBA}$

(prepared on the day)

(b) 3H4NBA

(prepared four months ago)

(c) 1,5-DAN

(prepared on the day)

(d) 1,5-DAN

(prepared four months ago)

\section{\%Int.}

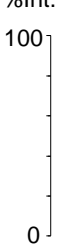

$\left.\mathrm{a}_{18}\right|_{18}$

$a_{14} a_{15} a_{16} a_{17}^{a_{18}}$

100
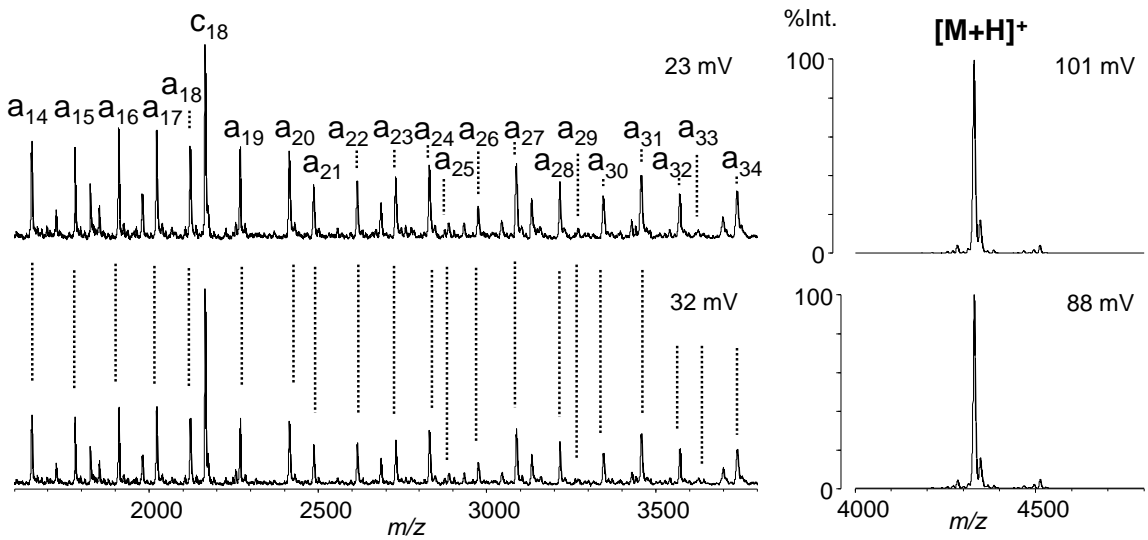

$1007 \quad 88 \mathrm{mV}$

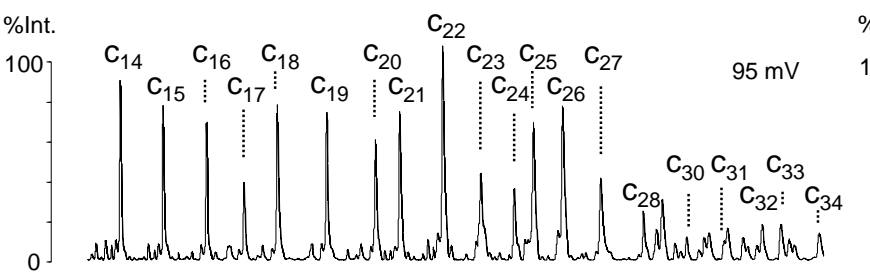

$\%$ Int.

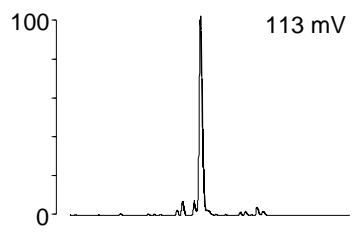

$-$

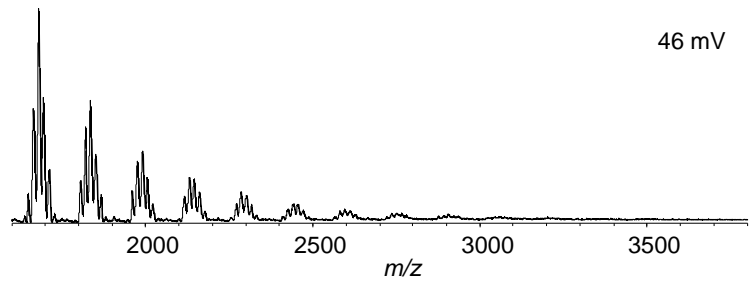

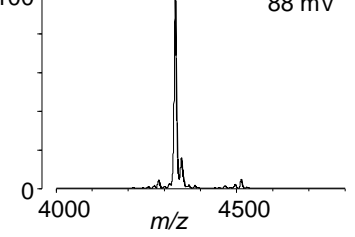

MS

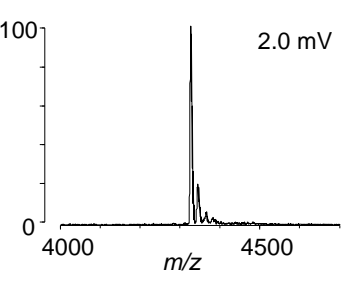

Figure S-2. MS and ISD spectra of amyloid $\beta 1-40$ using 3H4NBA and 1,5-DAN matrices solution prepared $(\mathrm{a}, \mathrm{c})$ on the day, or $(\mathrm{b}, \mathrm{d})$ four months ago, in linear positive-ion mode. 
3H4NBA

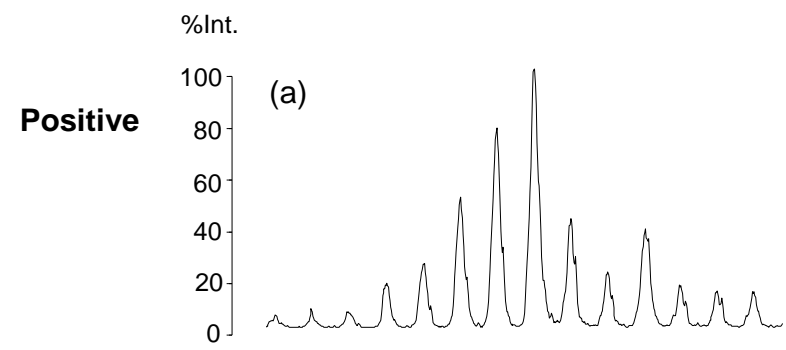

$[\mathrm{M}-\mathrm{H}]^{-}$

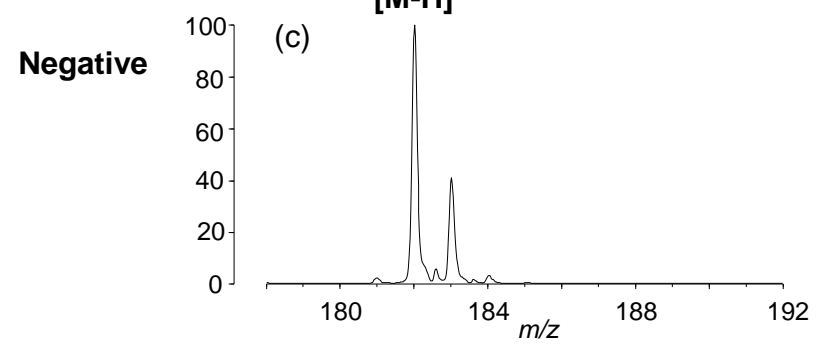

3H4NBA+analytes
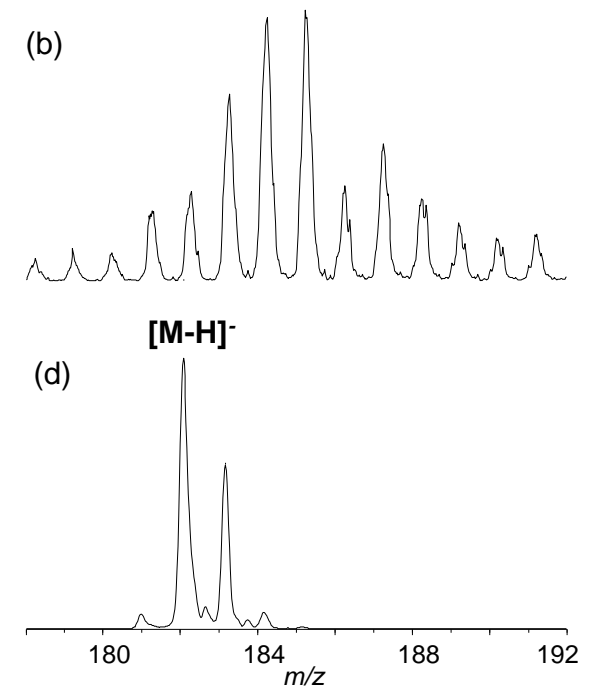

Figure S-3. Mass spectra of 3H4NBA matrix with or without analytes in $(a, b)$ positive- and $(c, d)$ negative-ion modes. 